\title{
The impact of previous therapy strategy on the efficiency of anlotinib hydrochloride as a third-line treatment on patients with advanced non-small cell lung cancer (NSCLC): a subgroup analysis of ALTER0303 trial
}

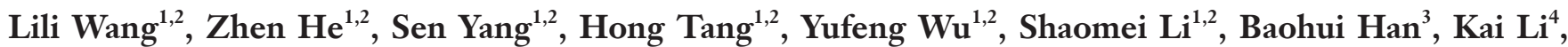 \\ Li Zhang ${ }^{5}$, Jianhua Shi ${ }^{6}$, Zhehai Wang ${ }^{7}$, Ying Cheng ${ }^{8}$, Jianxing He ${ }^{9}$, Yuankai Shi ${ }^{10}$, Weiqiang Chen ${ }^{11}$, \\ Yi Luo ${ }^{12}$, Lin $\mathrm{Wu}^{12}$, Xiuwen Wang ${ }^{13}$, Kejun $\mathrm{Nan}^{14}$, Faguang Jin ${ }^{15}$, Jian Dong ${ }^{16}$, Baolan $\mathrm{Li}^{17}$, Yan Sun ${ }^{10}$, \\ Qiming Wang ${ }^{1,2}$
}

${ }^{1}$ Oncology Department, Cancer Hospital Affiliated to Zhengzhou University, Zhengzhou 450008, China; ${ }^{2}$ Oncology Department, Henan Cancer Hospital, Zhengzhou 450008, China; ${ }^{3}$ Department of Pulmonary Medicine, Shanghai Chest Hospital, Shanghai Jiaotong University, Shanghai 200030, China; ${ }^{4}$ Department of Thoracic Oncology, Tianjin Medical University Cancer Hospital, Tianjin 300060, China; ${ }^{5}$ Department of Respiratory Diseases, Peking Union Medical College Hospital, Beijing 100032, China; ${ }^{6}$ Department of Oncology, Linyi Cancer Hospital, Linyi 276001, China; ${ }^{7}$ Department of Internal Medicine-Oncology, Shandong Cancer Hospital, Jinan 250117, China; ${ }^{8}$ Department of Thoracic Oncology, Jilin Cancer Hospital, Changchun 130012, China; ${ }^{9}$ Department of Thoracic Surgery, The First Affiliated Hospital of Guangzhou Medical University, Guangzhou 510120, China; ${ }^{10}$ Cancer Hospital Chinese Academy of Medical Sciences, Beijing 100021, China; ${ }^{11}$ Department of Pulmonary Medicine, Lanzhou Military General Hospital, Lanzhou 730050, China; ${ }^{12}$ Department of Medical Oncology, Hunan Cancer Hospital, Changsha 410006, China; ${ }^{13}$ Department of Chemotherapy, Qilu Hospital of Shandong University, Jinan 250012, China; ${ }^{14}$ Department of Oncology, The First Affiliated Hospital of Xi'an Jiaotong University, Xi'an 710061, China; ${ }^{15}$ Department of Respiratory Diseases, Tang Du Hospital, Xi'an 710038, China; ${ }^{16}$ First Department of Medical Oncology, Yunnan Cancer Hospital, Kunming 650118, China; ${ }^{17}$ Department of General Medicine, Capital Medical University, Beijing Chest Hospital, Beijing 101149, China

Contributions: (I) Conception and design: Q Wang; (II) Administrative support: Q Wang; (III) Provision of study materials or patients: All authors; (IV) Collection and assembly of data: All authors; (V) Data analysis and interpretation: L Wang, Q Wang; (VI) Manuscript writing: All authors; (VII) Final approval of manuscript: All authors.

Correspondence to: Dr. Qiming Wang. Oncology Department, Cancer Hospital Affiliated to Zhengzhou University, Zhengzhou 450008, China; Oncology Department, Henan Cancer Hospital, Zhengzhou 450008, China. Email: qimingwang1006@126.com.

Background: Lung cancer remains one of the deadliest cancers worldwide. The ALTER0303 trial revealed that anlotinib might be used as a third-line or further treatment in non-small cell lung cancer (NSCLC) patients. Meanwhile, the impact of previous therapy strategies on the efficiency of anlotinib still remains unknown.

Methods: The subgroup of patients in ALTER0303 were analyzed by using Kaplan-Meier estimates, Pearson $\chi^{2}$, or Fisher's exact test.

Results: There was no statistical significance on progression-free survival (PFS) and overall survival (OS) among patients in different previous antiangiogenic treatments groups. Patients in the chest radiotherapy (CRT) group had longer median PFS than the non-CRT group (5.93 vs. $4.63 \mathrm{~m}, \mathrm{P}=0.027$ ). Regardless of what kind of epidermal growth factor receptor tyrosine kinase inhibitors (EGFR TKI) and chemotherapy regimens were used previously, all patients gained longer PFS in the anlotinib group, while only patients treated with vinorelbine/platinum in the EGFR wild type group, pemetrexed/platinum, vinorelbine/ platinum, and gefitinib in the EGFR mutation group, and EGFR TKI used as the first line group could benefit from anlotinib on OS. When the OS was calculated from the time of diagnosis to the death, anlotinib could have increased median OS about 6 months (33.8 vs. $27.8 \mathrm{~m}, \mathrm{P}<0.001)$ compared to the placebo with a hazard ratio (HR) (95\% CI): 0.77 (0.60, 1.00). 


\begin{abstract}
Conclusions: This study indicated that previous bevacizumab or endostatin treatments had no impact on the efficiency of anlotinib. Patients with CRT history benefited more from anlotinib on PFS. EGFR TKI and chemotherapy treatment history had more impact on OS than PFS in patients treated with anlotinib compared to placebo.
\end{abstract}

Keywords: Non-small cell lung cancer (NSCLC); anlotinib; antiangiogenic therapy

Submitted Jan 22, 2019. Accepted for publication Sep 23, 2019.

doi: 10.21037/tlcr.2019.09.21

View this article at: http://dx.doi.org/10.21037/tlcr.2019.09.21

\section{Introduction}

Lung cancer is one of the highest incidences of cancers in the world. It represents $12.9 \%$ of the global cancer incident burden and correlates with around one-fifth of the cancer fatalities worldwide (1). Approximately $85 \%$ of patients with lung cancer are diagnosed as non-small cell lung cancer (NSCLC) (2). Although the 5-year survival rate in unresectable NSCLC has been greatly improved in recent years due to antiangiogenic treatment and the targeted treatment, according to driver-genes, NSCLC still remains the deadliest cancers in China (3). After receiving the standard first and second-line treatments, Chinese patients seldom have effective drugs to choose from in third-line treatment for further improving their overall survival (OS).

Anlotinib (AL3818) hydrochloride, a novel oral multitarget tyrosine kinase inhibitor (TKI), shows broad anticancer activity in many solid tumors both in vivo and in vitro (4-8). Anlotinib suppressed tumor angiogenesis and proliferation via blocking the receptor of tyrosine kinases in the signaling pathway of vascular endothelial growth factor receptor (VEGFR) 1 to 3, platelet-derived growth factor receptor (PDGFR) $\alpha$ and $\beta$, fibroblast growth factor receptor (FGFR) 1 to 4 , and stem cell factor receptor (7). In phase 3 of the randomized, double-blinded ALTER0303 clinical trial, anlotinib was used as a third-line or further treatment in patients with advanced NSCLC (stage IIIB to IV) (8). A total of 439 patients from 31 hospitals in China were enrolled in this trial, and 296 patients were randomized into the anlotinib group, and 143 were randomized into the placebo group. The primary endpoint of OS was observed significantly longer in the anlotinib group (median, 9.6 months; 95\% CI, 8.2-10.6) than the placebo group (median, 6.3 months; $95 \%$ CI, 5.0-8.1), with a hazard ratio (HR) of 0.68 (95\% CI, 0.54-0.87; P=0.002). Progression-free survival (PFS) was also improved significantly in the anlotinib group compared with the placebo group [median, 5.4 vs. 1.4 months; HR, 0.25 (95\% CI, 0.19-0.31); $\mathrm{P}<0.001$ ]. This clinical trial revealed that anlotinib had great efficacy and was well-tolerated as thirdline and further therapy among Chinese patients in this trial, indicating a potential treatment option for patients with advanced NSCLC.

Like most antiangiogenic drugs, the biomarker for anlotinib is still not very clear. What kind of patients would benefit from anlotinib treatment still remains unknown. In this study, we analyzed the subgroups data in phase 3 of ALTER 0303 clinical trial to evaluate whether different kinds of previous treatments will have an impact on the efficiency of anlotinib.

\section{Methods}

\section{Study design and treatment}

This double-blind, multicenter, randomized phase 3 clinical trial (ClinicalTrials.gov identifier: NCT02388919) was undertaken in 31 hospitals in China to estimate the efficacy and safety of anlotinib in patients with advanced NSCLC. The trial was conducted according to the principles of the Declaration of Helsinki and Good Clinical Practice requirements.

As reported previously (8), 439 patients were enrolled between March 1, 2015, and August 31, 2016. Inclusion criteria included the following: 18 to 75 years old; histological or cytological diagnosed NSCLC; pathologically confirmed as stage III and IV; Eastern Cooperative Oncology Group (ECOG) Performance status score 0 or 1 ; expected life of no less than 3 months; having at least one measurable lesion; disease progression after at least 2 lines of chemotherapy or at least 1 line of chemotherapy and TKI therapy for the patients with EGFR 
mutation or ALK rearrangement; adequate main organ function. Exclusion criteria included brain metastases that were uncontrolled or controlled for less than 2 months; central squamous lung cancer with the cavity; or hemoptysis $(>50 \mathrm{~mL} / \mathrm{d})$.

The primary endpoint was OS. The key secondary endpoints were PFS, objective response rate (ORR), disease control rate (DCR), and quality of life. The treatments of all patients before entering this trial have been well documented in detail.

\section{Procedures}

Patients were randomly divided into the anlotinib group or the placebo group by a 2:1 ratio. Anlotinib $(12 \mathrm{mg} / \mathrm{d})$ capsule or matched placebo was administered daily every continuous 14 days with 7 days break. Every 3 weeks were defined as one cycle of the treatment. The treatment terminated if patients had disease progression, toxicity intolerance, or withdrew the consent. The tumor was evaluated by computed tomography according to the Response Evaluation Criteria in Solid Tumors guidelines, version 1.1. Tumor assessment was performed within 2 weeks before treatment once per cycle during the first 2 cycles of the treatment and then assessed once every 2 cycles. The toxicity, efficacy, and survival of patients were followed up every 8 weeks until the death of the patient or until the data cutoff date (January 6, 2017), or whichever came first.

\section{Statistical analysis}

PFS was defined as the time from the date of randomizing to the date of disease progression. OS was defined as the time from the date of randomizing to the date of the patient's death. We used multivariable Cox proportional hazards models for PFS and OS. In model 1, we included 10 variables from the patient characteristics, plus previous antiangiogenic drugs. In model 2, we included the same variables as in model 1 but changed previous chemotherapy history to previous chemotherapy agents. In model 3, we included the same variables as in model 1 but changed the previous target therapy history to previously targeted agents. In model 4, we included the same variables as in model 1 but changed radiotherapy history to previous chest radiotherapy (CRT). Pearson $\chi^{2}$, or Fisher's exact test, was used to comparing the incidence of each group. All the statistical analysis was carried out on the basis of two side $\alpha=0.05$ and $95 \%$ CI by using SPSS 20.0 software. Median PFS and OS for patients were analyzed using Kaplan-Meier analysis.

\section{Results}

As described previously (8), a total of 439 patients were randomized in the ALTER0303 clinical trial (NCT02388919). Two patients withdrew their consent after randomization, and 294 patients ultimately remained in the anlotinib group, with 143 patients in the placebo group. It has been demonstrated that anlotinib could prolong PFS (5.37 vs. $1.40 \mathrm{~m} \mathrm{P<0.0001)} \mathrm{and} \mathrm{OS} \mathrm{(9.63} \mathrm{vs.} 6.30 \mathrm{~m}$ $\mathrm{P}=0.0018$ ) significantly as third-line therapy in patients with advanced NSCLC (8). Previous therapeutic strategies were well balanced in both the anlotinib group and placebo group except for tumor surgery history (Table 1).

In the anlotinib group, 11 patients used bevacizumab, and 16 patients used endostatin as the previous treatment before entering this clinical trial. We divided the patients who received anlotinib into three groups: the bevacizumab group (11 patients), endostatin group (16 patients), and without bevacizumab or endostatin group (268 patients). There was no statistical significance between PFS and OS among patients treated with anlotinib among the three groups (Figure 1): bevacizumab (11 patients), endostatin (16 patients), and without bevacizumab or endostatin group (268 patients). There were 13 patients with the best efficacy of both endostatin and anlotinib that could be evaluated in the endostatin group (Figure $2 A$ ), and 10 patients in the bevacizumab group with the valuable best efficacy of both bevacizumab and anlotinib (Figure 2B). After multivariable adjustment, previous antiangiogenic drugs were not associated with PFS and OS in patients with anlotinib (Tables $S 1, S 2)$. The best efficacy of endostatin, bevacizumab, and anlotinib is shown in Figure 2.

Sixty-one patients had a history CRT, and they had been treated with lung and/ or mediastinum radiotherapy before using anlotinib. Among these patients, 46 had a recorded dose of radiotherapy. The median months from the CRT to the treatment of anlotinib was 11.87 months (0.9267.93 months), while $52 \%$ (24/46) of patients received radical radiotherapy with $60-65$ Gy, while the other $48 \%$ (22/46) of patients received palliative radiotherapy with 30-50 Gy. As shown in Figure 3, patients in the CRT group had longer median PFS than the non-CRT group (5.93 vs. $4.63 \mathrm{~m}, \mathrm{P}=0.027$ ) with a hazard ratio (HR) that decreased to 0.68 (95\% CI, 0.52 to 0.96 ). However, no 
Table 1 Previous therapy strategy baseline of patients from ALTER0303 trial

\begin{tabular}{|c|c|c|c|}
\hline Subgroup & Placebo $(n=143)$ & Anlotinib $(n=294)$ & Comparison between groups \\
\hline No & $91(63.64)$ & $153(52.04)$ & Fisher's exact test $P=0.0241$ \\
\hline Yes & $52(36.36)$ & $141(47.96)$ & \\
\hline \multicolumn{4}{|l|}{ Chemotherapy } \\
\hline More than second line & $65(45.45)$ & $123(41.84)$ & \\
\hline Others (first line) & $0(0.00)$ & $4(1.36)$ & \\
\hline \multicolumn{4}{|l|}{ Chemotherapy regimens } \\
\hline Pemetrexed/platinum & $94(65.73)$ & 209 (71.09) & Fisher's exact test $P=0.2700$ \\
\hline Vinorelbine/platinum & $36(25.17)$ & $74(25.17)$ & Fisher's exact test $P=1.0000$ \\
\hline Gemcitabine/platinum & $77(53.85)$ & $156(53.06)$ & Fisher's exact test $\mathrm{P}=0.9188$ \\
\hline \multicolumn{4}{|l|}{ Radiotherapy } \\
\hline No & $78(54.55)$ & $176(59.86)$ & Fisher's exact test $P=0.3028$ \\
\hline Yes & $65(45.45)$ & $118(40.14)$ & \\
\hline \multicolumn{4}{|l|}{ Target therapy } \\
\hline No & $74(51.75)$ & $136(46.26)$ & Fisher's exact test $P=0.3080$ \\
\hline Yes & $69(48.25)$ & $158(53.74)$ & \\
\hline
\end{tabular}

A

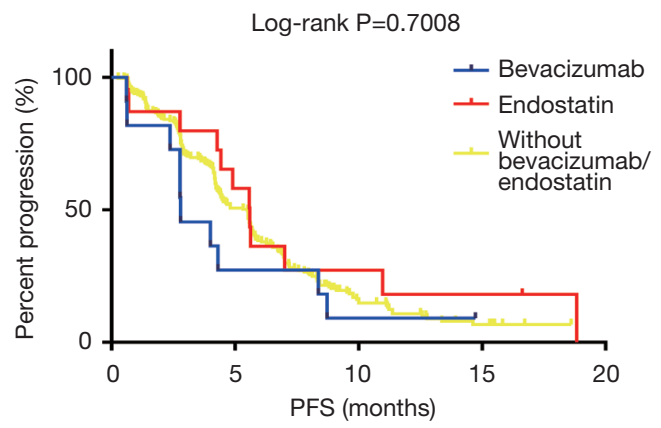

$\begin{array}{lccc}\text { Group } & \mathrm{N} \text { (events) } & \text { mPFS (months) } & \mathrm{P} \\ \text { Bevacizumab } & 11(10) & 2.80 & \\ \text { Endostatin } & 16(12) & 5.60 & 0.7008 \\ \text { Without bevacizumab/endostatin } & 267(191) & 5.50 & \end{array}$

B

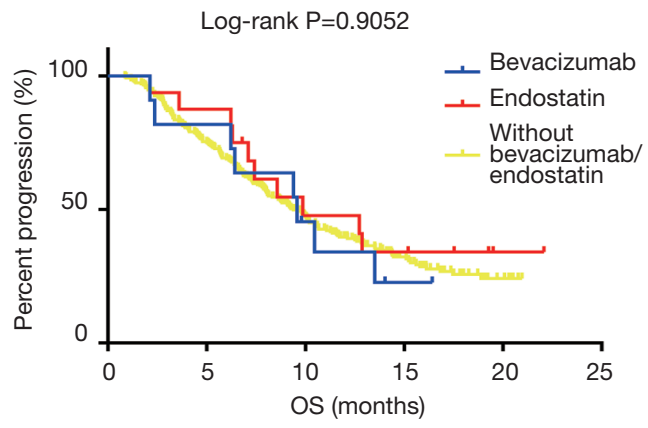

Group

Bevacizumab

Endostatin

Without bevacizumab/endostatin 267 (172)
$\mathrm{N}$ (events) mOS (months) P

$11(8) \quad 9.57$

$16(10) \quad 9.87$

0.9052

Figure 1 Kaplan-Meier estimates of overall and progression-free survival among different previous antiangiogenic therapy subgroup in patients treated with anlotinib. 

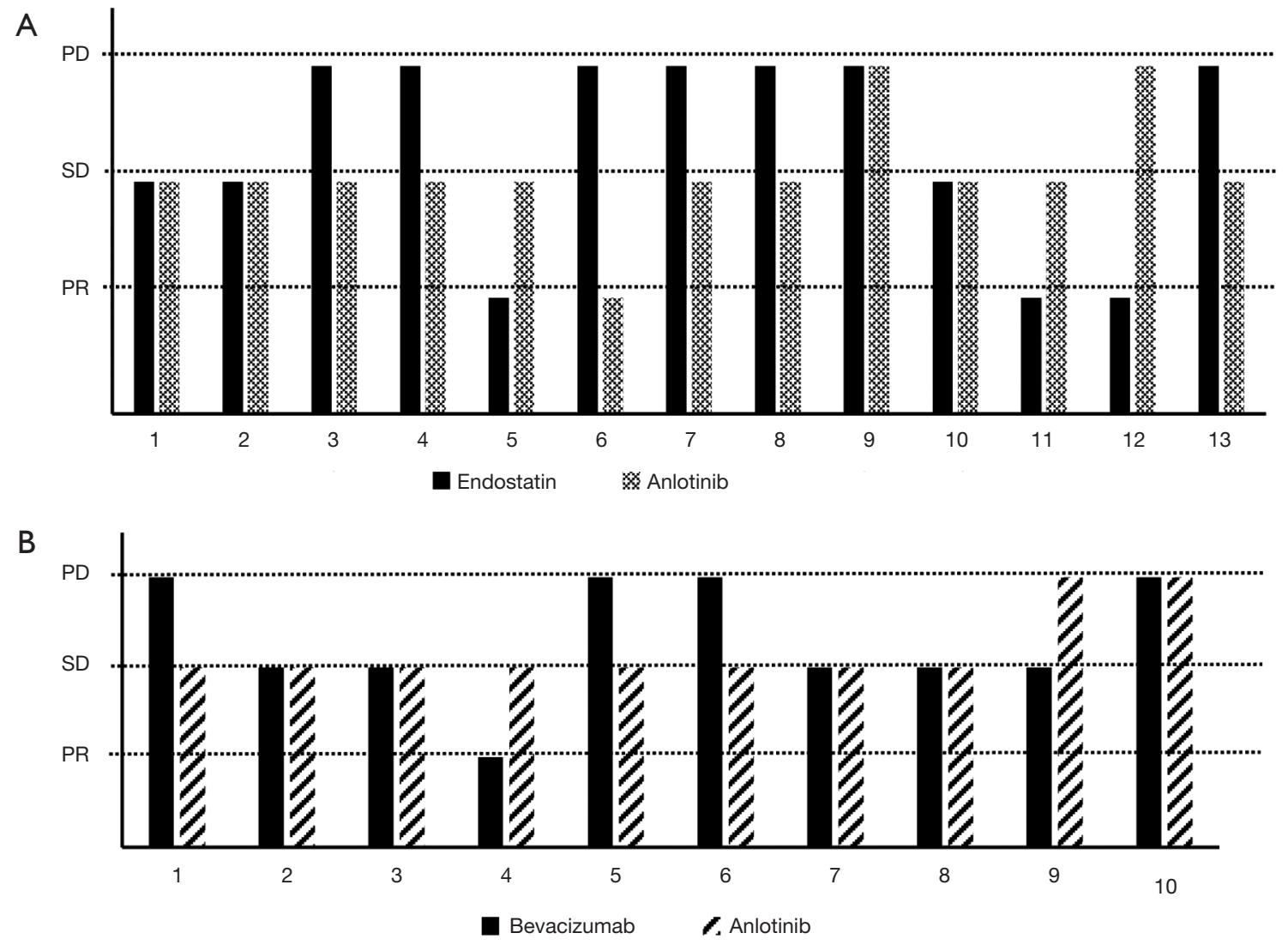

Figure 2 Comparison of efficiency between bevacizumab or endostatin and anlotinib in different patients.

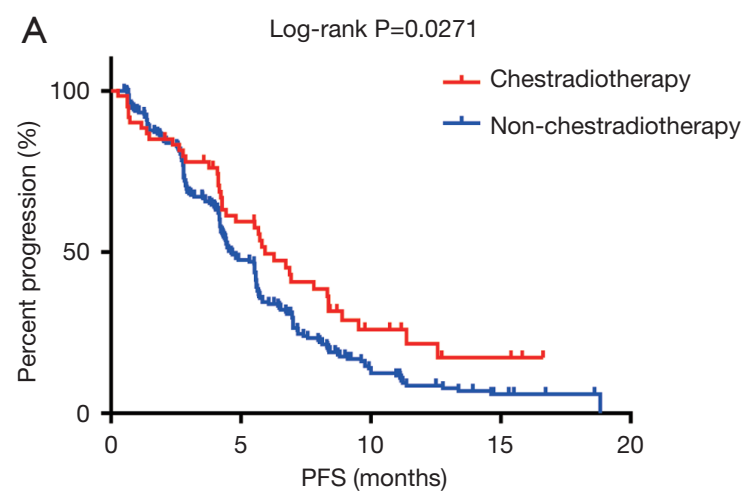

$\begin{array}{lccc}\text { Group } & \mathrm{N} \text { (events) } & \text { mPFS (months) } & \mathrm{P} \\ \text { Chestradiotherapy } & 61(40) & 5.93 & \\ \text { Non-chestradiotherapy } & 233(172) & 4.63 & 0.0271\end{array}$
B Log-rank $P=0.5375$

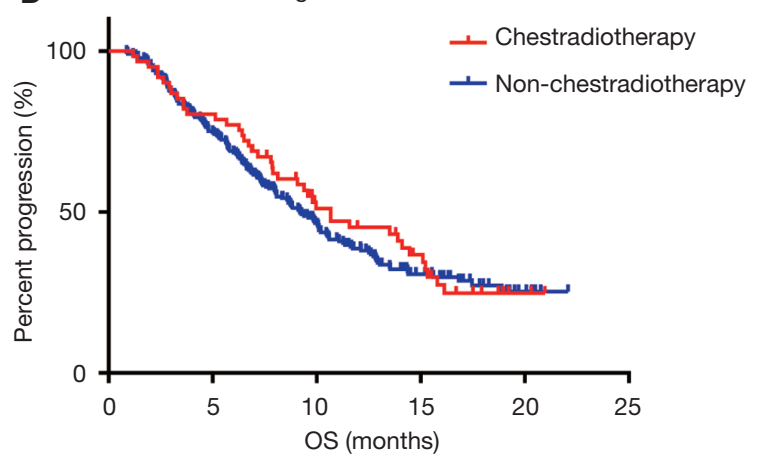

Group

Chestradiotherapy

Non-chestradiotherapy
$\mathrm{N}$ (events) mOS (months) $\quad \mathrm{P}$

$61(41) \quad 10.67$

$233(148) \quad 9.20$ 0.5375

Figure 3 Kaplan-Meier estimates of overall and progression-free survival between previous chest radiotherapy and non-chest radiotherapy subgroups in patients treated with anlotinib. 


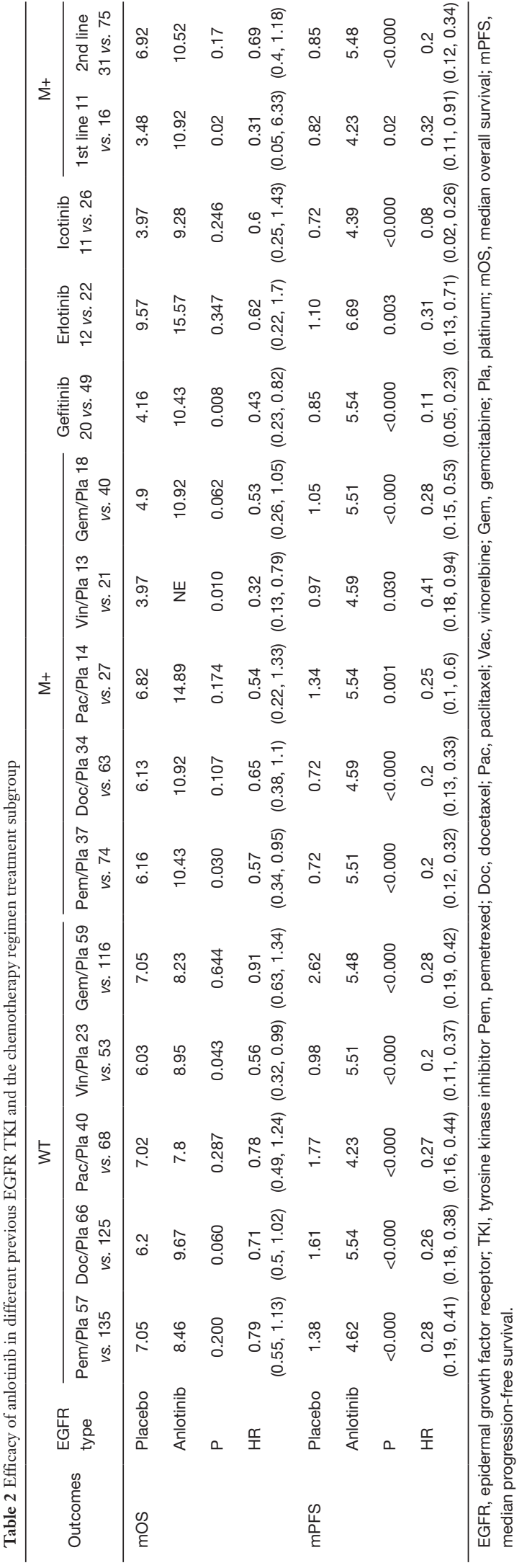

statistical significance was observed in OS data between the two groups. The median OS was 10.67 months (CRT group) and 9.20 months (non-CRT), respectively. After multivariable adjustment, CRT associated with longer PFS in patients with anlotinib, but it was associated with OS (Tables S3,S4).

We also analyzed whether previous chemotherapy or EGFR TKI treatment would have an impact on the efficacy of anlotinib. After multivariable adjustment, previous docetaxel plus platinum was associated with longer PFS in patients with anlotinib. However, previous chemotherapy regimens were associated with OS (Tables S5,S6). After multivariable adjustment, previous EGFR TKI was not associated with the PFS of patients with EGFR mutation in the anlotinib arm. However, the previous afatinib was associated with death, which might have been because of the small sample size $(\mathrm{n}=4)$ (Tables $S 7, S 8)$. As was shown in Table 2, patients were stratified by EGFR mutation, no matter what chemotherapy regimens patients had been treated with, patients from both the EGFR wild type group and the EGFR mutation group could benefit from anlotinib on PFS. Meanwhile, only patients treated with vinorelbine platinum $(\mathrm{P}=0.030)$ in the EGFR wild type group, pemetrexed platinum, and vinorelbine platinum $(\mathrm{P}=0.010)$ in the EGFR mutation group could benefit from anlotinib on OS. OS had statistical significance between the anlotinib group and placebo group when EGFR TKI used as the first-line therapy $(\mathrm{P}=0.020)$, but not for second-line therapy. Among the three EGFR TKIs, the gefitinib ( $\mathrm{P}=0.008)$, erlotinib, icotinib, and only OS in gefitinib $(\mathrm{P}=0.008)$ groups had statistical significance. All patients treated with any of the three EGFR TKIs as first-line or second-line therapy could benefit from anlotinib on PFS compared with the placebo.

\section{Discussion}

In recent years, antiangiogenic therapy has played an increasingly important role in treatments of advanced-stage NSCLC. Bevacizumab, a vascular endothelial growth factor (VEGF) monoclonal antibody, combined with platinumbased chemotherapy has been approved by FDA for use as standard first-line therapy in non-squamous NSCLC according to the results of phase 3 clinical trials (9). Bevacizumab greatly improved median OS from 10.3 to 12.3 months with an HR of 0.80 (95\% CI, 0.69-0.93), but in the third-line therapy, none of the previous multi-target TKIs, such as sorafenib (10), sunitinib (11), pazopanib (12) 
and vandetanib (13), could improve the OS times of patients compared to placebo. However, the ALTER0303 trial proved that anlotinib, a new third-line therapy choice in China, can benefit patients on both PFS and OS as reported previously. As multi-kinase inhibitors, sorafenib, sunitinib, and pazopanib target some receptor tyrosine and serine/threonine kinases, including receptors for a vascular endothelial growth factor (VEGFR), plateletderived growth factor (PDGFR), and c-Kit. Compare to these multikinase inhibitors, anlotinib has a wider range of targets, which might lead to better PFS and OS in patients with NSCLC. In this paper, the subgroup of previous therapeutic strategies analysis was undertaken to reveal further what kind of patients will benefit from anlotinib treatment.

As shown in Figure 2, 13 patients with endostatin treatment history and 10 patients with bevacizumab treatment history, the best efficacy of anlotinib does not correlate with the efficacy of previously used bevacizumab or endostatin. Among 7 patients who had PD effects of endostatin treatment, anlotinib therapy received $1 \mathrm{PR}, 4$ $\mathrm{SD}$, and $1 \mathrm{PD}$. Among 4 patients who had PD effects of bevacizumab treatment, anlotinib therapy received $3 \mathrm{SD}$ and $1 \mathrm{PD}$. As we know, cancer angiogenesis is regulated by several signaling pathways, including the vascular endothelial growth factor (VEGF), fibroblast growth factor (FGF) and platelet-derived growth factor (PDGF) pathways (14). In recent years, most anti-angiogenesis treatments have focused on the recombinant humanized monoclonal antibody, bevacizumab, which can block the VEGFR signaling pathway by binding to circulating VEGF (15). However, NSCLC cells often acquire resistance to bevacizumab following continued administration. Increasing evidence has indicated that the activation of the bypass signaling pathway, such as the FGF and PDGF signaling pathways, is the main acquired resistance mechanism to Bevacizumab therapy (16). Anlotinib blocks VEGFR 1 to 3 , PDGFR $\alpha$ and $\beta$, FGFR 1 to 4 , and c-KIT (7), while bevacizumab only combined with VEGF (17). Endostatin, a 20-KDa C-terminal fragment of collagen XVIII, inhibits vascular endothelial cell proliferation, and VEGF/VEGFR signal transduction (18). This may be due to these three antiangiogenic regimens having different targets, and even patients who did not benefit from bevacizumab or endostatin treatment can benefit from anlotinib in third-line therapy. However, the number of patients who had received prior bevacizumab and endostatin was very low in this clinical trial, and thus, the findings need to be verified in a larger sample of patients.

CRT is a common strategy to strengthen the stability of local chest tumors. In our study, patients with CRT previously might have had longer PFS times in the anlotinib treatment group, while CRT did not impact patients' OS time. Radiation therapy (RT) has been proven to have a role in the induction of the antitumor immune response (19). RT could promote increasing antigenic expression, releasing pro-inflammatory cytokine, which recruits immune cells, promoting antigen cross-presentation (20). Also, there is increasing evidence that anti-VEGF therapy might change the tumor microenvironment from an immunosuppressive to an immune permissive status (21). In phase III randomized trial, IMpower 150, the results showed a significant improvement in PFS and OS with the addition of atezolizumab to bevacizumab combined with chemotherapy as first-line treatment for non-squamous metastatic NSCLC (22). In this study, we found patients with CRT had a longer PFS. This may be due to CRT changing the immune microenvironment of cancer, which might increase the efficiency of anlotinib. However, this conclusion was drawn from a retrospective analysis in limited patients. It needs to be verified by prospective clinical research. Chemotherapy and EGFR TKI were the most common strategies used as the first- or secondline treatments in NSCLC patients. In this study, we also discovered that most of the previous EGFR TKI and different chemotherapy regimens have an impact on PFS rather than OS in the anlotinib group. When the previous treatment time was considered, anlotinib may increase OS time around 6 months compared to the placebo group.

\section{Conclusions}

This study revealed that previous bevacizumab or endostatin treatments had no impact on the efficacy of anlotinib in third-line treatment. Patients with CRT history seem to benefit more on PFS from anlotinib treatment but not for OS. Also, no matter what kind of EGFR TKI and chemotherapy regimens, all patients would gain longer PFS from anlotinib treatment. However, only patients treated with vinorelbine/platinum in the EGFR wild type group, pemetrexed/platinum, vinorelbine/platinum, and gefitinib EGFR mutation group, along with the EGFR TKI used as the first-line therapy group could benefit from anlotinib on OS compared to the placebo. These findings may help us to find patients who are suitable for anlotinib treatment according to their previous therapeutic strategies. Due to 
the limited number of patients in every subgroup, these results need to be further verified by a larger population.

\section{Acknowledgments}

We thank the patients and their families as well as the investigators and study teams for their participation in the study.

Funding: This work was supported by a grant from the Medical Science and Technology Foundation of Henan Province (No. 201601026) and in part by the National Natural Science Foundation of China (No. 81272600), Henan Provincial Training Abroad Foundation for Leaders of Medical Science (No. 201082), Henan Provincial Special Funds for Health and Technological Innovative Talents (No. 2011020155), Henan Provincial Research Program of Application Foundation and Advanced Technology (No. 112300410033), a project co-sponsored by the Henan Province and Ministry of Health of Medical Science and Technology Program (No. 201601026), the 51282 project Leading Talent of Henan Provincial Health Science and Technology Innovation Talents (No. [2016]32), and Wu Jieping Medical Foundation for Clinical Research (No. 320.6799.15018). It was also supported by the Program for Science and Technology Innovation Talents in Universities of Henan Province (No. 18HASTIT044). The funders had no role in study design, data collection, and analysis, decision to publish, or preparation of the manuscript.

\section{Footnote}

Conflicts of Interest: The authors have no conflicts of interest to declare.

Ethical Statement: The trial was conducted according to the principles of the Declaration of Helsinki and Good Clinical Practice requirements. All 31 institutions obtained approval from the research ethics board of each site. All the patients were willing to participate and signed informed consent. The authors are accountable for all aspects of the work in ensuring that questions related to the accuracy or integrity of any part of the work are appropriately investigated and resolved.

\section{References}

1. Ferlay J, Soerjomataram I, Dikshit R, et al. Cancer incidence and mortality worldwide: sources, methods and major patterns in GLOBOCAN 2012. Int J Cancer 2015;136:E359-86.

2. Miller KD, Siegel RL, Lin CC, et al. Cancer treatment and survivorship statistics, 2016. CA Cancer J Clin 2016;66:271-89.

3. She J, Yang P, Hong Q, et al. Lung cancer in China: challenges and interventions. Chest 2013;143:1117-26.

4. He C, Wu T, Hao Y. Anlotinib induces hepatocellular carcinoma apoptosis and inhibits proliferation via Erk and Akt pathway. Biochem Biophys Res Commun 2018;503:3093-9.

5. Xie C, Wan X, Quan H, et al. Preclinical characterization of anlotinib, a highly potent and selective vascular endothelial growth factor receptor-2 inhibitor. Cancer Sci 2018;109:1207-19.

6. Lin B, Song X, Yang D, et al. Anlotinib inhibits angiogenesis via suppressing the activation of VEGFR2, PDGFRbeta and FGFR1. Gene 2018;654:77-86.

7. Sun Y, Niu W, Du F, et al. Safety, pharmacokinetics, and antitumor properties of anlotinib, an oral multitarget tyrosine kinase inhibitor, in patients with advanced refractory solid tumors. J Hematol Oncol 2016;9:105.

8. Han B, Li K, Wang Q, et al. Effect of Anlotinib as a Third-Line or Further Treatment on Overall Survival of Patients With Advanced Non-Small Cell Lung Cancer: The ALTER 0303 Phase 3 Randomized Clinical Trial. JAMA Oncol 2018;4:1569-75.

9. Patel JD, Socinski MA, Garon EB, et al. PointBreak: a randomized phase III study of pemetrexed plus carboplatin and bevacizumab followed by maintenance pemetrexed and bevacizumab versus paclitaxel plus carboplatin and bevacizumab followed by maintenance bevacizumab in patients with stage IIIB or IV nonsquamous non-small-cell lung cancer. J Clin Oncol 2013;31:4349-57.

10. Paz-Ares L, Hirsh V, Zhang L, et al. Monotherapy Administration of Sorafenib in Patients With NonSmall Cell Lung Cancer (MISSION) Trial: A Phase III, Multicenter, Placebo-Controlled Trial of Sorafenib in Patients with Relapsed or Refractory Predominantly Nonsquamous Non-Small-Cell Lung Cancer after 2 or 3 Previous Treatment Regimens. J Thorac Oncol 2015;10:1745-53.

11. Scagliotti GV, Krzakowski M, Szczesna A, et al. Sunitinib plus erlotinib versus placebo plus erlotinib in patients with previously treated advanced non-small-cell lung cancer: a phase III trial. J Clin Oncol 2012;30:2070-8.

12. Weiss JM, Villaruz LC, Socinski MA, et al. A single-arm phase II trial of pazopanib in patients with advanced non- 
small cell lung cancer with non-squamous histology with disease progression on bevacizumab containing therapy. Lung Cancer 2014;86:288-90.

13. Natale RB, Thongprasert S, Greco FA, et al. Phase III trial of vandetanib compared with erlotinib in patients with previously treated advanced non-small-cell lung cancer. J Clin Oncol 2011;29:1059-66.

14. Ballas MS, Chachoua A. Rationale for targeting VEGF, FGF, and PDGF for the treatment of NSCLC. Onco Targets Ther 2011;4:43-58.

15. Crinò L, Metro G. Therapeutic options targeting angiogenesis in nonsmall cell lung cancer. Eur Respir Rev 2014;23:79-91.

16. Cascone T, Xu L, Lin HY, et al. The HGF/c-MET Pathway Is a Driver and Biomarker of VEGFR-inhibitor Resistance and Vascular Remodeling in Non-Small Cell Lung Cancer. Clin Cancer Res 2017;23:5489-501.

17. Li H, Takayama K, Wang S, et al. Addition of bevacizumab enhances antitumor activity of erlotinib

Cite this article as: Wang L, He Z, Yang S, Tang H, Wu Y, Li S, Han B, Li K, Zhang L, Shi J, Wang Z, Cheng Y, He J, Shi Y, Chen W, Luo Y, Wu L, Wang X, Nan K, Jin F, Dong J, Li B, Sun Y, Wang Q. The impact of previous therapy strategy on the efficiency of anlotinib hydrochloride as a third-line treatment on patients with advanced non-small cell lung cancer (NSCLC): a subgroup analysis of ALTER0303 trial. Transl Lung Cancer Res 2019;8(5):575-583. doi: 10.21037/tlcr.2019.09.21 against non-small cell lung cancer xenografts depending on VEGF expression. Cancer Chemother Pharmacol 2014;74:1297-305.

18. An J, Lv W. Endostar (rh-endostatin) versus placebo in combination with vinorelbine plus cisplatin chemotherapy regimen in treatment of advanced non-small cell lung cancer: A meta-analysis. Thorac Cancer 2018;9:606-12.

19. Teng F, Kong L, Meng X, et al. Radiotherapy combined with immune checkpoint blockade immunotherapy: Achievements and challenges. Cancer Lett 2015;365:23-9.

20. Kalbasi A, June CH, Haas N, et al. Radiation and immunotherapy: a synergistic combination. J Clin Invest 2013;123:2756-63.

21. Hegde PS, Wallin JJ, Mancao C. Predictive markers of anti-VEGF and emerging role of angiogenesis inhibitors as immunotherapeutics. Semin Cancer Biol 2018;52:117-24.

22. Socinski MA, Jotte RM, Cappuzzo F, et al. Atezolizumab for First-Line Treatment of Metastatic Nonsquamous NSCLC. N Engl J Med 2018;378:2288-301. 


\section{Supplementary}

Table S1 Multivariable Cox proportional hazards model 1 for PFS

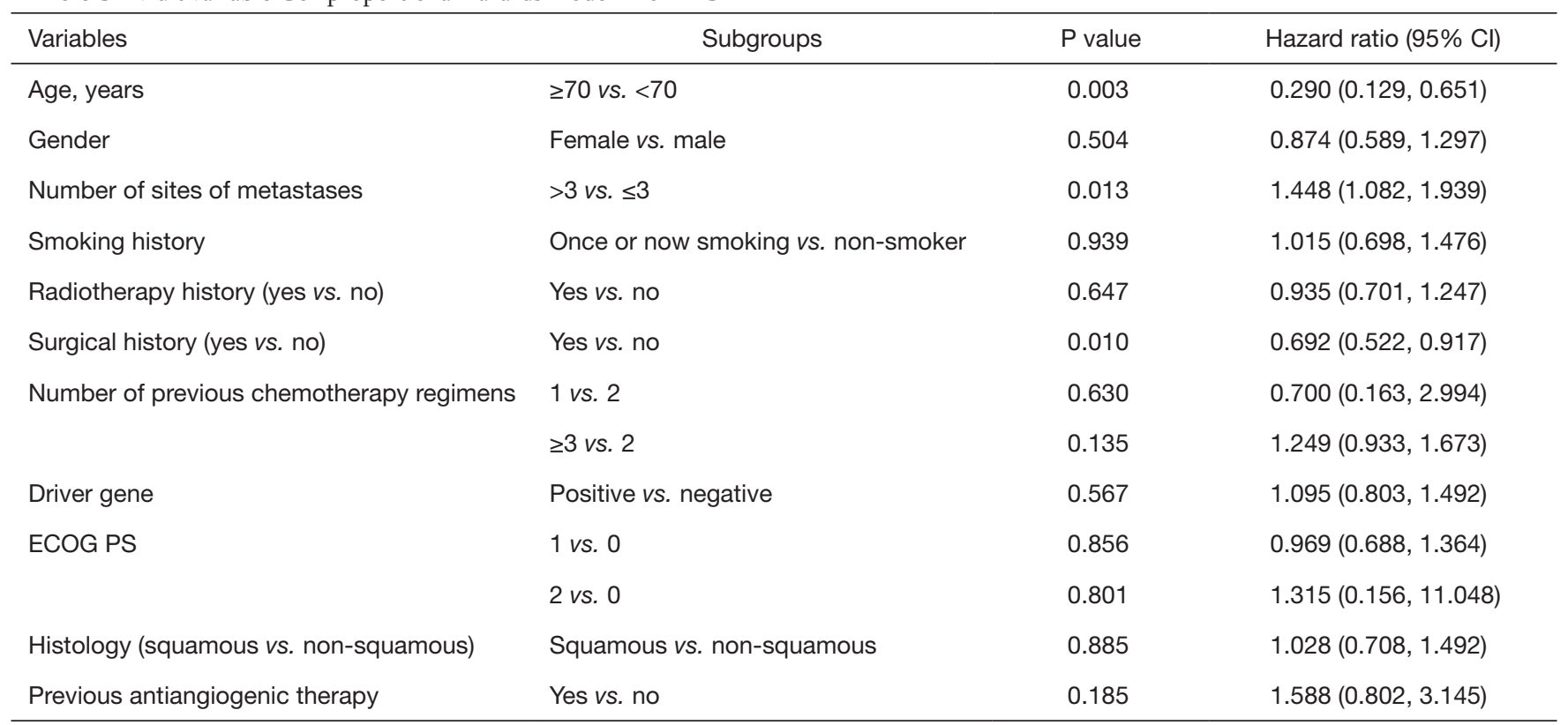

PFS, progression-free survival.

Table S2 Multivariable Cox proportional hazards model 1 for OS

\begin{tabular}{llll}
\hline Variables & \multicolumn{1}{c}{ Subgroups } & $\mathrm{P}$ value & $\mathrm{HR}(95 \% \mathrm{Cl})$ \\
\hline Age, years & $\geq 70$ vs. $<70$ & 0.076 & $0.483(0.216,1.079)$ \\
Gender & Female vs. male & 0.176 & $0.751(0.496,1.137)$ \\
Number of sites of metastases & $>3$ vs. $\leq 3$ & $<0.0001$ & $2.059(1.530,2.771)$ \\
Smoking history & Once or now smoking vs. non-smoker & 0.301 & $1.222(0.836,1.785)$ \\
Radiotherapy history & Yes vs. no & 0.765 & $1.046(0.778,1.408)$ \\
Surgical history & Yes vs. no & 0.057 & $0.750(0.558,1.009)$ \\
Number of previous chemotherapy regimens & 1 vs. 2 & 0.111 & $1.278(0.945,1.728)$ \\
& $\geq 3$ vs. 2 & 0.677 & $1.359(0.321,5.756)$ \\
Driver gene & Positive vs. negative & 0.112 & $0.758(0.539,1.066)$ \\
ECOG PS & 1 vs. 0 & 0.019 & $1.630(1.085,2.448)$ \\
& 2 vs. 0 & 0.084 & $4.313(0.824,22.569)$ \\
Histology & Squamous vs. non-squamous & 0.560 & $0.889(0.599,1.319)$
\end{tabular}

OS, overall survival. 
Table S3 Multivariable Cox proportional hazards model 4 for PFS

\begin{tabular}{llll}
\hline Variables & \multicolumn{1}{c}{ Subgroups } & P value & HR $(95 \% \mathrm{Cl})$ \\
\hline Age, years & $\geq 70$ vs. $<70$ & 0.003 & $0.290(0.130,0.646)$ \\
Gender & Female vs. male & 0.572 & $0.893(0.603,1.322)$ \\
Number of sites of metastases & $>3$ vs. $\leq 3$ & 0.012 & $1.446(1.084,1.930)$ \\
Smoking history & Once or now smoking vs. non-smoker & 0.990 & $1.002(0.690,1.457)$ \\
Previous chest radiotherapy & Yes vs. no & 0.034 & $0.639(0.422,0.967)$ \\
Surgical history & Yes vs. no & 0.006 & $0.672(0.506,0.892)$ \\
Number of previous chemotherapy regimens & $\geq 3$ vs. 2 & 0.205 & $1.209(0.902,1.620)$ \\
& 1 vs. 2 & 0.566 & $0.653(0.153,2.797)$ \\
ECOG PS & 1 vs. 0 & 0.668 & $0.927(0.657,1.309)$ \\
& 2 vs. 0 & 0.670 & $1.584(0.191,13.109)$ \\
Histology & Squamous vs. non-squamous & 0.632 & $1.096(0.754,1.594)$ \\
Driver gene & Positive vs. negative & 0.630 & $1.079(0.792,1.470)$ \\
Previous antiangiogenic therapy & Yes vs. no & 0.253 & $1.495(0.751,2.975)$
\end{tabular}

PFS, progression-free survival.

Table S4 Multivariable Cox proportional hazards model 4 for OS

\begin{tabular}{llll}
\hline Variables & \multicolumn{1}{c}{ Subgroups } & P value & $\mathrm{HR}(95 \% \mathrm{Cl})$ \\
\hline Age, years & $\geq 70$ vs. $<70$ & 0.734 & $0.787(0.197,3.136)$ \\
Gender & Female vs. male & 0.799 & $1.097(0.540,2.229)$ \\
Number of sites of metastases & $>3$ vs. $\leq 3$ & $<0.001$ & $2.537(1.545,4.166)$ \\
Smoking history & Once or now smoking vs. non-smoker & 0.352 & $1.338(0.725,2.467)$ \\
Previous chest radiotherapy & Yes vs. no & 0.524 & $0.845(0.503,1.419)$ \\
Surgical history & Yes vs. no & 0.606 & $0.884(0.555,1.410)$ \\
Number of previous chemotherapy regimens & $\geq 3$ vs. 2 & 0.723 & $1.089(0.679,1.749)$ \\
& 1 vs. 2 & 0.243 & $3.705(0.412,33.348)$ \\
ECOG PS & 1 vs. 0 & 0.325 & $1.341(0.748,2.403)$ \\
& 2 vs. 0 & 0.266 & $3.241(0.409,25.705)$ \\
Histology & Squamous vs. non-squamous & 0.919 & $1.036(0.525,2.044)$ \\
Driver gene & positive vs. negative & 0.169 & $0.692(0.409,1.170)$ \\
Previous antiangiogenic therapy & Yes vs. no & 0.721 & $1.224(0.403,3.715)$
\end{tabular}

OS, overall survival. 
Table S5 Multivariable Cox proportional hazards model 2 for PFS

\begin{tabular}{|c|c|c|c|}
\hline Variables & Subgroups & $\mathrm{P}$ value & $\mathrm{HR}(95 \% \mathrm{Cl})$ \\
\hline Gender & Female vs. male & 0.579 & $0.893(0.598,1.334)$ \\
\hline Number of sites of metastases & $>3$ vs. $\leq 3$ & 0.007 & $1.507(1.121,2.025)$ \\
\hline Smoking history & Once or now smoking vs. non-smoker & 0.765 & $1.060(0.725,1.549)$ \\
\hline Surgical history & Yes vs. no & 0.024 & $0.720(0.542,0.957)$ \\
\hline Driver gene & Positive vs. negative & 0.669 & $1.071(0.782,1.466)$ \\
\hline \multirow[t]{2}{*}{ ECOG PS } & 1 vs. 0 & 0.734 & $0.942(0.668,1.329)$ \\
\hline & 2 vs. 0 & 0.677 & $1.569(0.189,13.055)$ \\
\hline Docetaxel + platinum & Yes vs. no & 0.022 & $0.702(0.519,0.949)$ \\
\hline Paclitaxel + platinum & Yes vs. no & 0.568 & $1.093(0.806,1.482)$ \\
\hline Vinorelbine + platinum & Yes vs. no & 0.270 & $0.830(0.596,1.156)$ \\
\hline Gemcitabine + platinum & Yes vs. no & 0.582 & $0.915(0.668,1.255)$ \\
\hline Previous antiangiogenic therapy & Yes vs. no & 0.272 & $1.457(0.744,2.854)$ \\
\hline
\end{tabular}

PFS, progression-free survival.

Table S6 Multivariable Cox proportional hazards model 2 for OS

\begin{tabular}{|c|c|c|c|}
\hline Variables & Subgroups & $P$ value & $\mathrm{HR}(95 \% \mathrm{Cl})$ \\
\hline Gender & Female vs. male & 0.227 & $0.774(0.512,1.172)$ \\
\hline Number of sites of metastases & $>3$ vs. $\leq 3$ & $<0.0001$ & $2.124(1.567,2.878)$ \\
\hline Smoking history & Once or now smoking vs. non-smoker & 0.212 & $1.274(0.871,1.863)$ \\
\hline Surgical history & Yes vs. no & 0.023 & $0.707(0.524,0.953)$ \\
\hline Driver gene & Positive vs. negative & 0.117 & $0.762(0.543,1.070)$ \\
\hline \multirow[t]{2}{*}{ ECOG PS } & 1 vs. 0 & 0.028 & $1.582(1.050,2.383)$ \\
\hline & 2 vs. 0 & 0.076 & $4.500(0.854,23.726)$ \\
\hline Docetaxel + platinum & Yes vs. no & 0.060 & $0.739(0.540,1.012)$ \\
\hline Paclitaxel + Platinum & Yes vs. no & 0.612 & $0.917(0.658,1.280)$ \\
\hline Vinorelbine + platinum & Yes vs. no & 0.124 & $0.755(0.528,1.080)$ \\
\hline Gemcitabine + platinum & Yes vs. no & 0.367 & $1.164(0.837,1.618)$ \\
\hline Previous antiangiogenic therapy & Yes vs. no & 0.883 & $1.058(0.500,2.236)$ \\
\hline
\end{tabular}

OS, overall survival. 
Table S7 Multivariable Cox proportional hazards model 3 for PFS

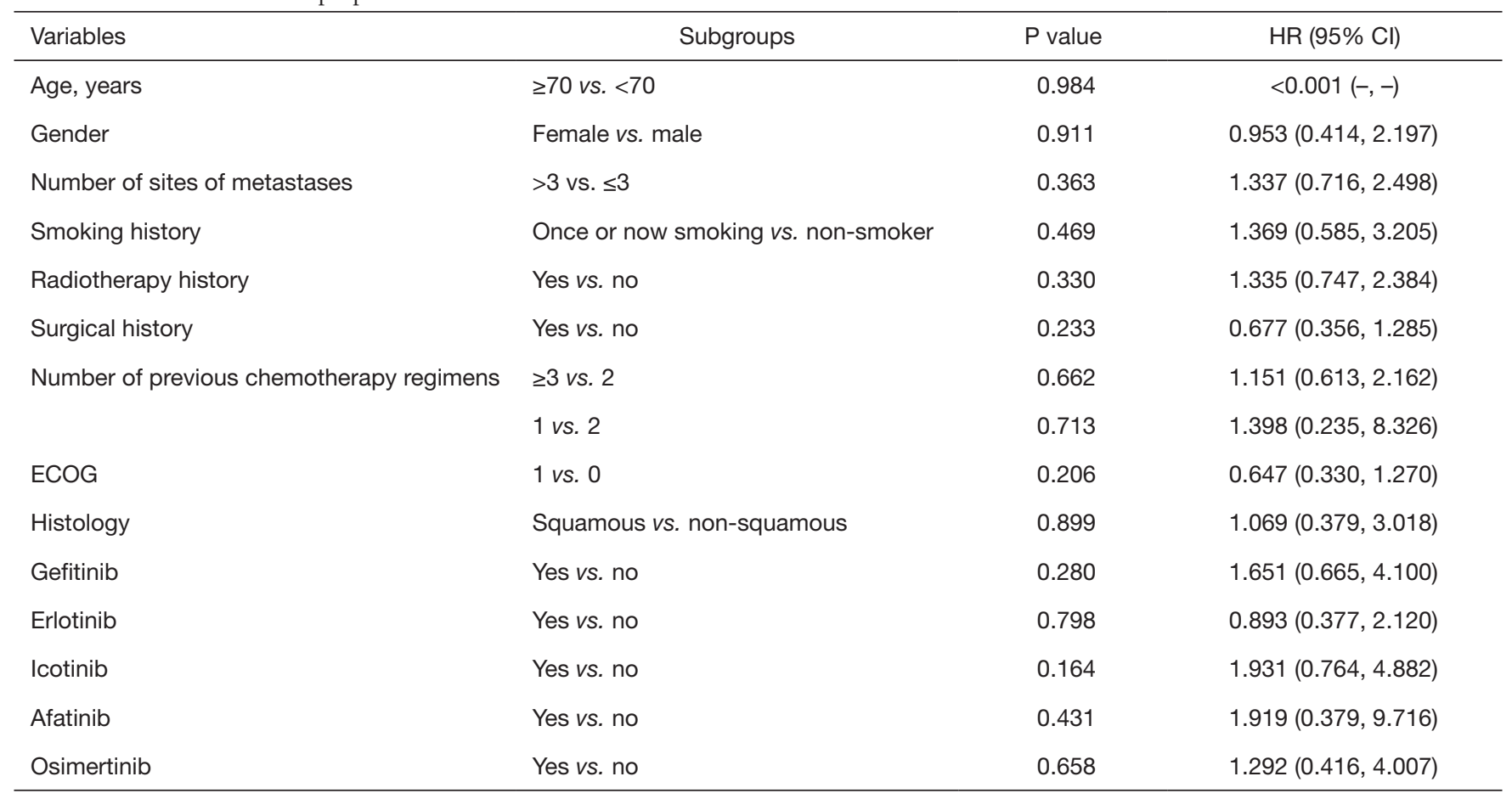

PFS, progression-free survival.

Table S8 Multivariable Cox proportional hazards model 3 for OS

\begin{tabular}{|c|c|c|c|}
\hline Variables & Subgroups & $P$ value & $\mathrm{HR}(95 \% \mathrm{Cl})$ \\
\hline Gender & Female vs. male & 0.004 & $0.267(0.109,0.654)$ \\
\hline Number of sites of metastases & $>3$ vs. $\leq 3$ & 0.001 & $2.980(1.539,5.770)$ \\
\hline Smoking history & Once or now smoking vs. non-smoker & 0.717 & $0.862(0.387,1.921)$ \\
\hline Surgical history & Yes vs. no & 0.592 & $1.185(0.636,2.209)$ \\
\hline \multirow[t]{2}{*}{ Number of previous chemotherapy regimens } & $\geq 3$ vs. 2 & 0.022 & $2.339(1.131,4.837)$ \\
\hline & 1 vs. 2 & 0.307 & $2.368(0.454,12.359)$ \\
\hline ECOG & 1 vs. 0 & 0.140 & $1.843(0.818,4.155)$ \\
\hline Erlotinib & Yes vs. no & 0.209 & $0.568(0.235,1.372)$ \\
\hline Icotinib & Yes vs. no & 0.393 & $1.558(0.563,4.317)$ \\
\hline Afatinib & Yes vs. no & 0.032 & $5.573(1.156,26.881)$ \\
\hline Osimertinib & Yes vs. no & 0.777 & $0.856(0.293,2.507)$ \\
\hline
\end{tabular}

OS, overall survival. 